\title{
Understanding the Plant Immune System
}

\author{
Fumiaki Katagiri and Kenichi Tsuda \\ Department of Plant Biology, Microbial and Plant Genomics Institute, University of Minnesota, 1500 Gortner Ave., \\ St. Paul 55108, U.S.A.
}

Submitted 29 April 2010. Accepted 12 July 2010.

Plant immunity is controlled by a complex signaling network. Here, we discuss how the complexity of the network affects our views and approaches in studying the plant immune network. We propose that the mode of plant immunity is mainly determined by how the shared signaling network is used rather than by a signaling machinery specific to each mode, that balancing the robustness of immunity and the negative effect of immunity on plant fitness is a key driver in evolution of the immune network, that comparisons of plant mutant to wild-type phenotypes may not be very effective in elucidating the underlying signaling mechanisms, and that mechanistic understanding of the network can improve our ability to predict the performance of immunity.

Distinct uses of an integrated signaling network rather than distinct networks.

Pattern-triggered immunity (PTI) and effector-triggered immunity (ETI) are two modes of plant immunity defined by the types of molecules recognized by plants as indicators of a pathogen attack (Jones and Dangl 2006). Recognition of molecular patterns characteristic of microbes (microbe-associated molecular patterns [MAMPs]) by plant pattern-recognition receptors (PRR), which are typically localized in the plant cell membrane, triggers PTI (Boller and Felix 2009). Recognition of pathogen effectors by plant resistance $(\mathrm{R})$ proteins, which are typically localized inside the plant cell, triggers ETI (Jones and Dangl 2006). Although the characteristics of immunity associated with PTI and ETI are different, many molecular responses are associated with both PTI and ETI (Abramovitch et al. 2006; Navarro et al. 2004; Tao et al. 2003; Zipfel et al. 2006). The latter fact suggests overlap in the signaling machinery between PTI and ETI.

Our recent study (Tsuda et al. 2009) demonstrated that at least some cases of PTI and ETI-namely, PTI triggered by the bacterial flagellin fragment flg22 (flg22-PTI) and ETI triggered by the Pseudomonas syringae effector AvrRpt2 (AvrRpt2-ETI)—depend on common elements of the signaling machinery. Both flg22-PTI and AvrRpt2-ETI were mostly abolished in the Arabidopsis quadruple mutant dde2/ein2/ pad4/sid2. DDE2, EIN2, and SID2 are required for jasmonate (JA) biosynthesis (Park et al. 2002), most of the ethylene (ET) response (Alonso et al. 1999), and salicylate (SA) biosynthesis in response to pathogens (Wildermuth et al. 2001), respectively. PAD4 affects SA synthesis and expression of many SAindependent defense-responsive genes (Glazebrook et al. 2003;

Corresponding author: F. Katagiri; E-mail: katagiri@umn.edu
Jirage et al. 1999). Because the signaling mechanisms in which these genes function as hubs significantly interact with each other, we prefer to call them signaling sectors of a network rather than signaling pathways, as the term "pathways" implies relative independence. Thus, four signaling sectors, the JA, ET, $\mathrm{SA}$, and PAD4 sectors, are absent in the quadruple mutant. We also showed that the way the four sectors interact was very different in flg22-PTI and AvrRpt2-ETI: synergistic and compensatory, respectively. If our observations can be generalized, major differences between PTI and ETI result not from differences in the nature of the signaling machinery but from differences in how the common signaling machinery is used.

One of our observations appears to contradict such a generalization: whereas AvrRpt2-ETI was highly dependent on the four-sector network, AvrRpm1-ETI was not (Tsuda et al. 2009). Is this an indication that different signaling networks are required in different cases of ETI? We speculate that major parts of the networks are not different. Rather, we propose that the speed of the responses affects which immune responses provide effective immunity. It is known that the spectra of the responses are very similar between AvrRpm1-ETI and AvrRpt2-ETI but that responses are substantially faster in AvrRpm1-ETI (Ritter and Dangl 1996; Tao et al. 2003). Here, for the sake of discussion, we hypothetically name the relatively early and late ETI responses the phase I and II responses. We postulate that, in AvrRpm1-ETI, phase I is initiated earlier, and effective phase I responses mask potential roles of phase II responses controlled by the four-sector network. Although the same phase I responses are triggered in AvrRpt2-ETI, they probably occur too late to be effective. Therefore, the phase II responses controlled by the four-sector network are important in AvrRpt2-ETI. This kinetic difference can cause apparent differences in requirements for signaling mechanisms. Again, we argue that major differences are not caused by different signaling networks but mainly by differences in how the same network is used. The only difference between the case of AvrRpm1-ETI and AvrRpt2-ETI and the case of AvrRpt2-ETI and flg22-PTI is that the signaling network in question needs to be expanded to include phase I-controlling signaling sectors as well as the phase II-controlling four sectors.

How can the same network be used differently? We suggest that, after perception of pathogen attack by either a PRR or an $\mathrm{R}$ protein, the signal branches into multiple signal flows and that multiple signals enter from different points of the common network. The common network can be considered as circuit computing based on these multiple inputs. There must be differences in the strength or timing of the multiple input signals between PTI and ETI. It is easy to imagine that such differences in the input signal set can lead to different ways of signal processing in the network. 
In addition to PTI and ETI acting against the hemibiotrophic pathogen $P$. syringae pv. tomato DC3000, the four-sector signaling network is important in immunity against the necrotrophic fungus Alternaria brassicicola (Tsuda et al. 2009). Therefore, it appears that plants use an integrated signaling network for various modes of immunity but use the common network differently for different modes.

\section{PTI and ETI are complementary.}

Hasn't PTI been beaten by well-adapted pathogens, which deliver effectors into the plant cell to interfere with PTI signal-
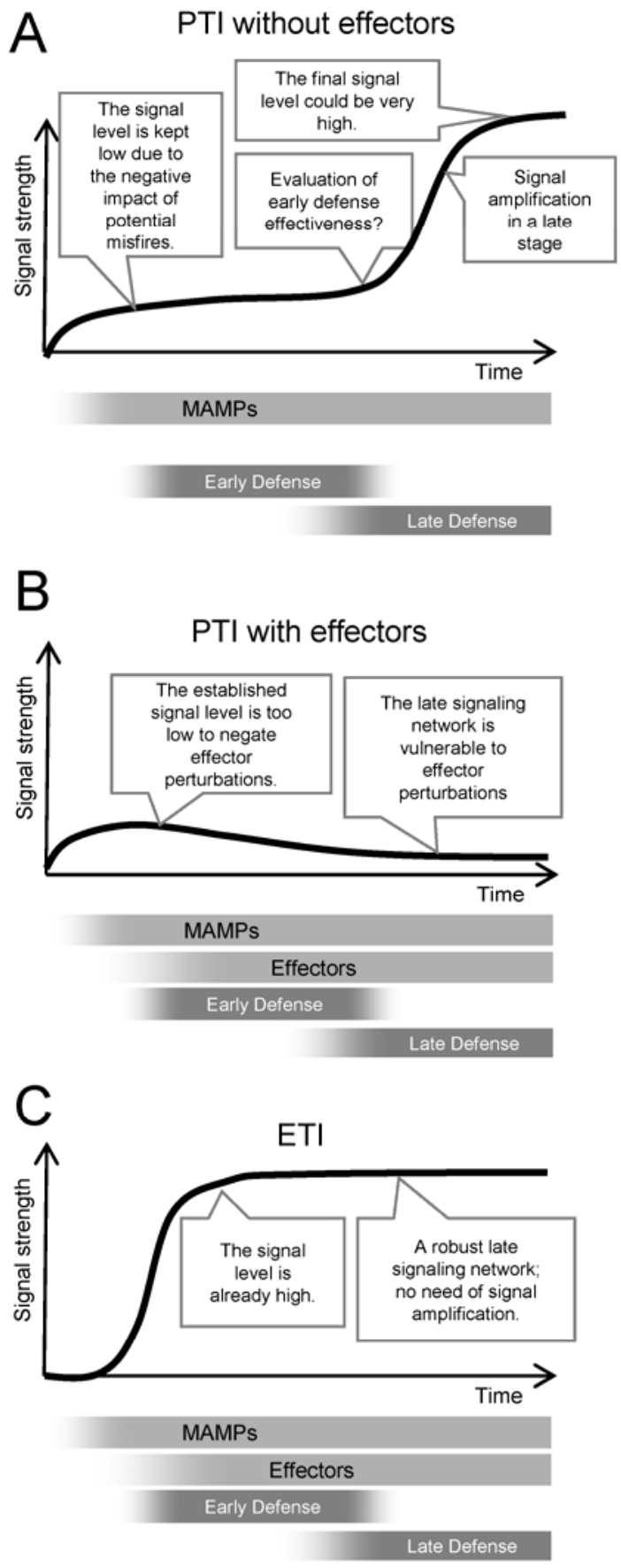

Fig. 1. Kinetics of immune signaling defines pattern-triggered immunity (PTI) and effector-triggered immunity (ETI) and their interactions with effectors (conceptual diagrams). A, PTI without effector interference. B, PTI with effector interference. C, ETI. In each panel, timing of delivery of microbe-associated molecular patterns (MAMPs) and effectors from pathogens and early and late defense responses in plants are indicated below the graph. ing? If so, how could ETI be effective against well-adapted pathogens while using the same signaling network? We propose that the negative impact of immune responses on plant fitness and the specificity of pathogen recognition, in addition to the speed of immune responses, are the keys to answer these questions. Immune responses are generally resource-expensive (Bolton 2009) and deleterious to plants. Consider the hypersensitive response (HR), which results in plant cell death. Plants must be subject to selection for avoidance of unnecessary immune responses. Balancing points between necessary and unnecessary immune responses are affected by the specificity of pathogen recognition.

Molecular patterns that trigger PTI are conserved among pathogens and benign microbes (Boller and Felix 2009). If strong immune responses were activated rapidly without distinguishing pathogens from benign microbes, the responses would be excessive in many cases and carry fitness costs. Therefore, PTI responses have likely been selected for weakness in early stages due to the low specificity in pathogen recognition (Fig. 1A). This possible requirement for a slow start suggests that enhancing PTI in crop plants in a nonspecific manner may not be beneficial. Under experimental conditions, PTI-enhanced plants may only be tested with pathogens, revealing enhanced immunity without much fitness cost. However, in the field where many benign microbes are present, the plants may suffer substantial fitness costs.

In addition to the possible requirement for a slow start in PTI, we speculate that plants have a way to evaluate the effectiveness of early PTI responses (Fig. 1A). For example, continuous or increasing MAMP signaling may be interpreted as an inadequacy of early responses. If the early responses are sufficient, plants can abort further, unnecessary immune responses. If the early responses are not sufficient, plants may use the four-sector network to amplify the signal for stronger responses in a later stage. This signal amplification likely involves positive feedback loops involving multiple sectors (Shah 2003); hence, synergistic interactions among the sectors were observed with PTI (Tsuda et al. 2009). If plants are given a sufficient lead time, PTI can be potent. This is demonstrated by the strong immunity triggered by treatment of plants with flg22 1 day prior to inoculation with virulent $P$. syringae (Tsuda et al. 2009; Zipfel et al. 2004). In such experiments, the flg22 concentration and the number of plant cells responding are likely much higher than under physiological conditions. This could be interpreted by the plant as an indication that the early weak response is not sufficient, resulting in a late surge mediated by the four-sector network.

Two notions about PTI, the requirement for a slow start and the late-stage synergistic sector interactions, can explain why PTI is vulnerable to attack by adapted pathogens (Fig. 1B). A slow start of PTI can give adapted pathogens opportunities to effectively interfere with PTI signaling: initial low concentrations of effectors can easily suppress weak PTI signaling. The late signaling sectors are vulnerable to effector perturbations because the synergistic interactions among the sectors can be disrupted by inactivation of one of the sectors.

In contrast to MAMPs, effectors are hallmarks of potent pathogens, not benign microbes; therefore, plants have likely been selected to activate strong immune responses in ETI immediately after recognition by an $\mathrm{R}$ protein of a very low concentration of the corresponding effector (Fig. 1C). Because plants rapidly generate strong signals, it is difficult for a low concentration of effectors to interfere with ETI signaling in phase I. Because the signal is already strong, the signal does not need to be amplified in phase II. Instead, the phase II signaling sectors, which highly overlap with the late stage sectors in PTI, can be used for network compensation. Consequently, impair- 
ment of some signaling sectors by pathogen effectors do not have a large impact on overall immunity. Thus, we speculate that ETI signaling was selected to be robust against perturbations by pathogen effectors based on speed in phase I and network compensation in phase II. Such robustness built into the network properties and dynamics is important because pathogens evolve much faster than plants; therefore, rapid changes in the effector repertoire can alter the points at which the signaling network is perturbed.

A need to balance strengths of immunity and its negative impacts may occur in some cases of ETI, although to a lesser extent than in PTI. AvrRpm1 appears to be a more potent effector than AvrRpt2 (Kim et al. 2005). Probably, responses in AvrRpm1-ETI were selected to be faster than those in AvrRpt2-ETI to negate the more potent effector rapidly. However, this strong immunity comes with a higher fitness cost because $R P M 1$, which is the $R$ gene for AvrRpm1, is associated with a large cost of resistance (Tian et al. 2003). Existence of slower AvrRpt2-ETI suggests that ETI can also start somewhat slowly to have an opportunity to evaluate the effectiveness of the phase I responses. The potato $R$ gene $R x$ confers extreme resistance against Potato virus $X$ : virus propagation is completely blocked without an HR (Bendahmane et al. 1999). However, when the viral coat protein alone, which is recognized by $\mathrm{Rx}$, is artificially expressed in an $R x^{+}$plant, an HR ensues (Bendahmane et al. 1999). If there is evaluation of the effectiveness of the very early response, this could be explained as follows: a default ETI program is rapidly aborted when virus propagation is completely blocked by very early responses; however, continuous expression of the viral coat protein is interpreted by the plant as ineffectiveness of very early responses, and the HR, a later response which is more damaging to the plant but a more potent immune response, is activated.

What are the differences between PRR and $\mathrm{R}$ proteins? There are two aspects: different types of molecules that are recognized, molecular patterns and effectors; and different modes of immune responses to be triggered, weak in the beginning (PTI-like) and fast and strong (ETI-like). However, classifications made according to these two aspects do not always coincide. For example, the rice Xa21 protein was initially classified as an $\mathrm{R}$ protein because it triggers ETI-like responses but it has recently been shown that it recognizes a molecular pattern (Lee et al. 2009). In addition, Xa21 is a receptor-like kinase with extracellular leucine-rich repeats, which suggests a common evolutionary origin with canonical PRR, such as FLS2, and a common ancestral signaling network for $\mathrm{Xa} 21$ and canonical PRR. An important point is that the molecular pattern recognized by $\mathrm{Xa} 21$ is specific to the Xanthomonas genus (Lee et al. 2009) (i.e., this molecular pattern is closely associated with pathogens). This example of Xa21 supports the notion that PTI-like and ETI-like responses are not mediated by distinct signaling networks but by different ways of using a common network that are selected based on how well the recognized molecules discriminate between pathogens and benign microbes.

Furthermore, if it is indeed true that the speed or strength of signaling is a major difference between PTI and ETI, we need to be cautious in designing experiments and interpreting their results in terms of PTI and ETI. For example, it has been shown that ectopic expression of some effectors in the plant can inhibit ETI (Block et al. 2008). However, this inhibitory effect may not be significant if the effectors are delivered at physiological concentrations with physiological timing.

The specificity of pathogen recognition has two sides. Due to high recognition specificity, ETI signaling evolved to be robust against pathogen effectors. However, pathogens often escape from ETI by evading such specific recognition. Due to the low recognition specificity, PTI cannot be very effective against well-adapted pathogens but it can provide good immunity with low cost on fitness against potential pathogens that are not well adapted. Thus, PTI and ETI have different strengths and weaknesses and are complementary in plant immunity.

\section{A sector-switching network.}

Does the network compensation in ETI mean that the sectors in the network are simply functionally redundant? We posit that the network is more sophisticated than that. For example, both the SA and JA sectors can positively contribute to AvrRpt2-ETI. However, the SA and JA sectors typically antagonize each other (Glazebrook 2005), which implies that only one of them can be highly activated at a given time. Such antagonistic regulatory relationships between sectors in the plant immune signaling network appear very common (Sato et al. 2010). It is likely that only a small number of sectors are highly activated at a given time and suppress other sectors when the network is intact. When such primary sectors are impaired by effector perturbations, secondary sectors, which can also positively contribute to immunity, are released from suppression and back up the functions of the primary sectors. When the secondary sectors are also impaired, the tertiary sectors kick in, and so on. We call this property of the network "sector switching." By having backups, immunity can be highly tolerant to effector perturbations. Using only a limited number of sectors at a given time can restrict the spectrum or strength of immune responses, which reduces the negative impact of the immune response on plant fitness. Thus, a sector-switching network can balance robustness in immunity and plant fitness, and we propose that the plant immune signaling network has such a sector-switching property under particular conditions, such as when ETI is triggered.

Sector switching may be influenced by conditions other than effector perturbations. For example, the levels of hormones involved in the signaling network can be affected by environmental conditions and life history, causing variation in initial conditions of the network before pathogen attack. With different initial conditions, the network may respond differently to the same inputs from pathogen attack. With a sector-switching network, the difference could be qualitative: different sectors could become primary sectors. This may lead to identification of different sectors as primary sectors in immunity triggered by the same molecule under different experimental conditions, such as in different laboratories. Similarly, different sectors might be identified as primary sectors in closely related plant species in which the network structure is largely conserved. An important point is that, although sector switching may occur relatively easily, the overall performance of immunity does not change much: immunity is robust although the underlying signaling flow may not be very stable.

\section{Dissociation between the "genotype-phenotype" paradigm and the physical mechanism.}

In simple signaling networks, such as mostly diverging networks or networks consisting of relatively independent signaling pathways, the function of a network component correlates well with the opposite of the phenotype observed in a loss-offunction mutant lacking the component. However, in a complex network, which contains multiple significant converging points, such as feedback and feedforward points, this may not be the case. In fact, this is what we observed with the plant immune signaling network.

We used a signaling allocation analysis to estimate the effect of each signaling sector and interactions among them as additive effects on a particular case of immunity (Tsuda et al. 2009). For 
example, AvrRpt2-ETI is mostly abolished in the quadruple mutant; therefore, we considered this as a (almost) no-immunity, wiped-out state. In a triple mutant, dde2/pad4/sid2, only the ET sector among the four sectors is functional; therefore, the immunity gained in the triple mutant compared with the quadruple mutant is allocated as the contribution of the ET signaling sector. Similarly, the contribution of each of the other signaling sectors was allocated using the other triple mutants. Including the data with the double mutants allowed us to allocate the parts of immunity that cannot be explained by the additive effect of two sectors to the two-sector interactions. Similarly, allocations to the three-sector and four-sector interactions were determined by including the data with the single mutants and the wild type. Thus, this conceptual "wipe-out and reconstitute stepwise" procedure used in the signaling allocation analysis allowed us to determine the mechanistic relationships among the sectors (Fig. 2, closed bars).

We define the genotype-phenotype paradigm as the assumption that the opposite of the mutant phenotype represents the function of the gene corresponding to the mutation (Fig. 2, open bars). For example, because AvrRpt2-ETI was reduced in the $d d e 2$ mutant by $0.3 \log _{10}$ unit compared with the wild type, the contribution of the JA sector, which is defined by DDE2, to the immunity is considered to be $0.3 \log _{10}$ unit according to the genotype-phenotype paradigm. The contributions of each sector and their interactions to AvrRpt2-ETI, determined by the signaling allocation analysis and according to the genotypephenotype paradigm, are very different (Fig. 2). For example, in AvrRpt2-ETI, the PAD4 sector can have a stronger contribution to immunity than the JA sector in the signaling allocation. However, the level of AvrRpt2-ETI lost was very similar between dde 2 and pad4; therefore, according to the genotypephenotype paradigm, the PAD4 and JA sectors appear to contribute equally to the immunity. The two-sector interactions look very different between the signaling allocation and the genotype-phenotype paradigm. The signaling allocation shows that most of the two-sector interactions are negative, while the genotype-phenotype paradigm shows that the two-sector interactions are positive or insignificant.

Why do they look so different? This is because the mutation in a sector not only removes the function of the sector but also removes all the interactions involving this sector (two-sector, three-sector, and four-sector interactions). Therefore, when sector interactions are significant (i.e., when a network is complex), the signaling mechanisms involved in the network cannot be accurately inferred based on the genotype-phenotype paradigm.

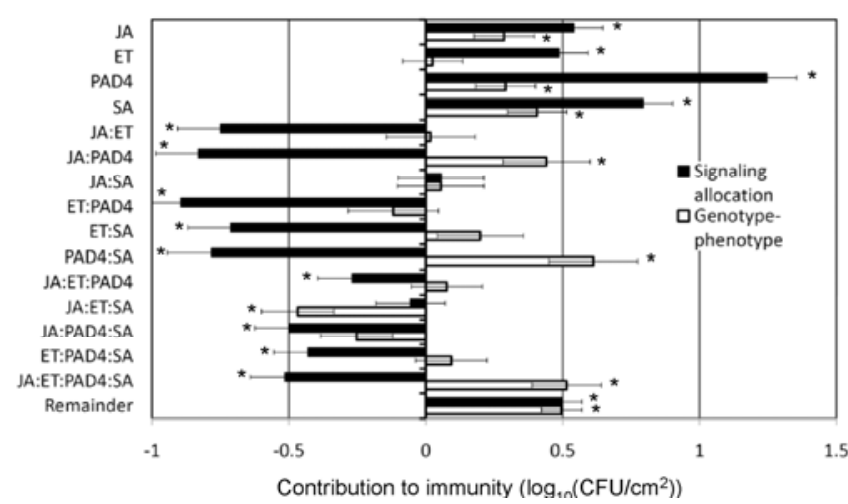

Fig. 2. Contributions of the four signaling sectors and their interactions in AvrRpt 2 effector-triggered immunity (ETI) as determined by signaling allocation analysis (closed bars) or mutant phenotypes (open bars; "Genotypephenotype"). Error bars, standard error; asterisk, $P<0.05$. JA = jasmonate, $\mathrm{ET}=$ ethylene, and $\mathrm{SA}=$ salicylate. The raw data used to generate this figure are from Tsuda and associates (2009).
In fact, the four-sector network in AvrRpt2-ETI is robust against not only pathogen attack but also genetic perturbations: without starting with the (almost) wiped-out state, it is very difficult to gain mechanistic information. This is called an identifiability problem of a robust system and provides a good example of the dissociation between the genotype-phenotype paradigm and the actual physical mechanisms in a complex network.

Due to the possible dissociation between the genotype-phenotype paradigm and the physical mechanism in a complex signaling network, we need to be cautious in mechanistic interpretations of mutant phenotypes. The "wipe out and reconstitute stepwise" approach demonstrates that studying the phenotype of a highly impaired genotype (e.g., the quadruple mutant in AvrRpt2-ETI) is an effective way to elucidate the mechanisms. This fact presents a new opportunity for forward genetics. For example, a dde2/ein $2 /$ sid2 triple mutant has almost intact AvrRpt2-ETI while the quadruple mutant, which has a pad4 mutation in addition, has lost most of AvrRpt2-ETI (Tsuda et al. 2009). This suggests that the robustness of the network is highly impaired in $d d e 2 / e i n 2 / s i d 2$, although the immunity phenotype is barely affected. A mutant screen using dde2/ein2/ sid2 as a parent line may identify loci that are involved in AvrRpt2-ETI in this impaired network state, in addition to mutations in pad4, rps 2 , and other genes whose mutations are known to impair AvrRpt2-ETI. Identification of causal mutations in such a complex genetic background has been quite challenging because mapping of the causal mutations has required crossing to a polymorphic line: the other line typically does not have the same starting mutations as the parent line (e.g., dde2/ein $2 /$ sid 2 in the above example), resulting in a complex segregation pattern; the other line may have unknown genetic differences that affect the phenotype of interest. However, advances in deep-sequencing technologies are making identification of causal mutations by sequencing realistic in Arabidopsis: sequencing of bulk segregants after multiple backcrossing of a mutant of interest to the parent line will quickly identify a small number of candidate mutations. Therefore, forward genetic screens, starting with genetic backgrounds in which the robustness of the network is highly reduced, together with deep-sequencing technologies, will facilitate identification of new components in a complex signaling network.

But, doesn't the genotype-phenotype paradigm drive evolution? Don't alterations in one or a few genes affect phenotypes and count in selection? True, at least for evolution in a relatively short time scale. Then, why do we need to know the mechanisms underlying a complex network other than for scientific curiosity? First, mechanistic properties of the network could influence evolution over a longer time scale. For example, a population of plants can accumulate genetic variation in the components of a robust network, such as the AvrRpt2-ETI signaling network, without much effect on the phenotype the network controls. In other words, the robustness of the network provides a buffering function to genetic variation in the network components, which is analogous to the capacitor function of HSP90 (Rutherford and Lindquist 1998). Accumulation of large variation within a plant population could increase the chance that the species survives after an environmental change, such as emergence of a new pathogen, because some subpopulations may have variations that are advantageous in the new environment. Second, mechanistic information is needed to make better predictions about network behavior.

\section{Predicting the behavior of a complex system over broad ranges of parameters.}

One major goal of plant sciences is to improve crop production: increase production while reducing environmental impacts of agriculture. Considering the future security of the food sup- 
ply, the potential of biofuels, and deterioration of natural environments, it is urgent to make leaps of improvement in crop production. Regarding plant immunity in the light of this goal, we need to find better balancing points between effectiveness of immunity and negative impacts of immunity in particular environments, such as different levels of pressure from various pathogens and existence of benign microbes. This can be conceptualized as the fitness landscape in particular environments (Fig. 3). For the sake of visualization, only two network parameters (e.g., concentrations of network components) are used in the figures. It should be noted that many more network parameters are likely to be important in the fitness landscape of an actual network. A complex network likely has a complex landscape (Fig. 3). The peaks indicated by arrows 1 through 4 are local optima in the fitness level. We want to know the combinations of parameter values that achieve such optimal fitness for plant improvement.

According to the selective pressures that a particular population has experienced through the course of evolution, the parameter value sets available in the population are likely trapped in one local optimum domain (Fig. 3A, the region enclosed by a dashed oval for local optimum 1). Due to the robustness of the network, this local optimum domain for the plant immune network may be relatively large compared with other networks but still very small compared with the entire parameter space. Within this domain, a strictly statistical model may work relatively well: an arbitrary function, such as a linear model and an artificial neural network, is fitted to observed parameter value-fitness level relationships; the fitted function is used to predict (i.e., approximate) the unknown fitness level for particular parameter values. This is feasible because observations of the fitness levels for dense parameter value sets within the domain can be made; such parameter value sets are naturally available in the form of genetic variants in the population. Note that, in a strictly statistical model, no mechanistic information about the immune network is utilized. In an environment very different from what the population has experienced during the history of the population evolution, such as modern agricultural environments, the landscape could be substantially altered (Fig. 3B). The local optimum 1 may shift to local optimum $1^{\prime}$. This is still within the same local optimum domain, and a strictly statistical model could predict the local optimum $1^{\prime}$ because observations within this domain can be made in the new environment with naturally available parameter value sets. However, other local optima, optima 3 and 4, may have substantially higher fitness levels than local optimum $1^{\prime}$. These are the optima we need to discover to make leaps of improvement in plant fitness. Once we know the parameter set values for such optima better than the evolutionarily selected optimum domain, we could engineer the plant for the parameter set values to achieve one of the better optima. Because parameter value sets available as natural variation are confined to the domain of local optimum $1^{\prime}$, it is not feasible to make observations with parameter value sets near local optima 3 and 4. An arbitrary function fitted for the domain of local optimum 1' cannot make accurate predictions for the parameter values near local optima 3 and 4 by extrapolation. Therefore, a combination of strictly statistical models and naturally available parameter value sets cannot predict the existence of local optima 3 and 4.

What about experimental exploration of the parameter space? It is not realistic to randomly explore outside the domain experimentally because the number of parameter value sets to explore increases exponentially as the number of parameters increase. For example, exploring five values for each of four parameters means making measurements for $5^{4}=$ 625 parameter value sets. In an actual network, the number of parameters to be explored is likely many more than four. Therefore, we need an in silico model to efficiently explore the vast parameter space computationally to make predictions about which parameters are important and what value sets for the important parameters result in local optima that are different from the one selected through evolution. When strictly statistical models are not appropriate for this purpose, what types of in silico model should we use?

One possible type of model that can be used for predictions outside the local optimum domain is a strictly mechanistic model that is built bottom-up based on the complete network structure and all the parameter values for the network components and links. For example, if a component is an enzyme, we need to know its concentration at the site, the Michaelis constant $\left(K_{m}\right)$, the maximum reaction rate $\left(V_{\max }\right)$, and other parameters such as the inhibitor constant $\left(K_{i}\right)$ for each related compound existing at the site. If the concentration of the enzyme is regulated during the process of interest, the regulatory process needs to be modeled in, too. If such complete mechanistic information were available, this type of model would make accurate predictions. The weakness of this approach is that the accuracy of the prediction precipitously deteriorates when the mechanistic information available is not quite complete, and we will not have complete information regarding the plant immune network any time soon.

Therefore, we need to seek a middle ground, a hybrid of statistical and mechanistic models, in which an approximating function is structurally constrained by all the mechanistic information available. With a structurally constrained approximating function, extrapolation outside the evolutionarily selected local optimum domain will have better accuracy. The distance from the current domain at which such a hybrid model can make good predictions depends on the amount of important mechanistic information and the complexity level of the parameter value-fitness level relationship observations used to create the model. Importantly, prediction accuracy of the model improves steadily as more mechanistic information and more parameter value-fitness level relationship observations become available. We suggest that this hybrid model approach is the most efficient, considering better predictions in a large part of the parameter space as the return on investment in collecting mechanistic information and parameter value-fitness level relationship observations.

To vastly improve crop production under particular environmental conditions, we need to explore local optima of fitness other than the one evolutionarily selected in a large parameter space. Making accurate predictions in a large part of the parameter space is the goal of systems biology. To achieve this goal with a complex network, it is essential to elucidate physi-
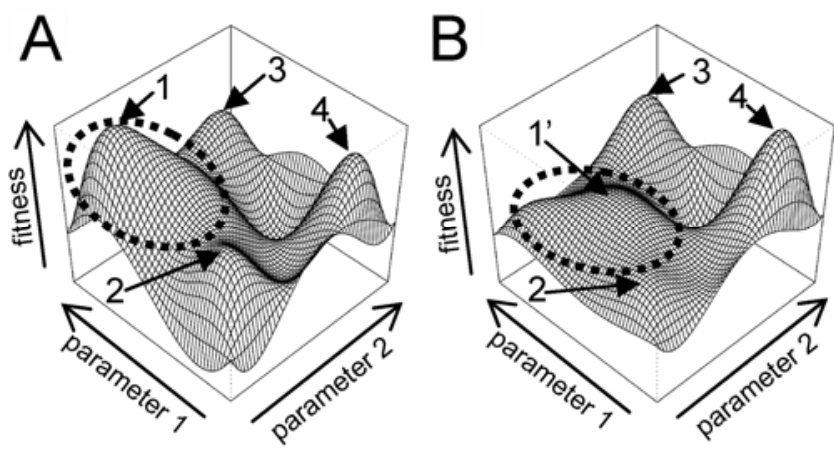

Fig. 3. Fitness landscape in the network parameter space in two different environments (conceptual diagrams). Local optima of fitness are indicated by arrows and numbers. Region encircled by a dotted curve indicates the domain of the evolutionarily selected local optimum. 
cal mechanisms underlying the network as well as to collect observations with various network parameter value sets. Gaining an ability to make accurate predictions regarding plant immunity in a large parameter space is what understanding the plant immune system means.

\section{ACKNOWLEDGMENTS}

We thank J. Glazebrook for critical reading of the manuscript and G. Bethke for her comments. This work was supported by the grant MCB0918908 to F. Katagiri from the National Science Foundation.

\section{LITERATURE CITED}

Abramovitch, R. B., Anderson, J. C., and Martin, G. B. 2006. Bacterial elicitation and evasion of plant innate immunity. Nat. Rev. Mol. Cell. Biol. 7:601-611.

Alonso, J. M., Hirayama, T., Roman, G., Nourizadeh, S., and Ecker, J. R. 1999. EIN2, a bifunctional transducer of ethylene and stress responses in Arabidopsis. Science 284:2148-2152.

Bendahmane, A., Kanyuka, K., and Baulcombe, D. C. 1999. The Rx gene from potato controls separate virus resistance and cell death responses. Plant Cell 11:781-792.

Block, A., Li, G., Fu, Z. Q., and Alfano, J. R. 2008. Phytopathogen type III effector weaponry and their plant targets. Curr. Opin. Plant Biol. 11:396-403.

Boller, T., and Felix, G. 2009. A renaissance of elicitors: perception of microbe-associated molecular patterns and danger signals by patternrecognition receptors. Annu. Rev. Plant Biol. 60:379-406.

Bolton, M. D. 2009. Primary metabolism and plant defense-fuel for the fire. Mol. Plant-Microbe Interact. 22:487-497.

Glazebrook, J. 2005. Contrasting mechanisms of defense against biotrophic and necrotrophic pathogens. Annu. Rev. Phytopathol. 43:205-227.

Glazebrook, J., Chen, W., Estes, B., Chang, H. S., Nawrath, C., Metraux, J. P., Zhu, T., and Katagiri, F. 2003. Topology of the network integrating salicylate and jasmonate signal transduction derived from global expression phenotyping. Plant J. 34:217-228.

Jirage, D., Tootle, T. L., Reuber, T. L., Frost, L. N., Feys, B. J., Parker, J. E., Ausubel, F. M., and Glazebrook, J. 1999. Arabidopsis thaliana PAD4 encodes a lipase-like gene that is important for salicylic acid signaling. Proc. Natl. Acad. Sci. U.S.A. 96:13583-13588.

Jones, J. D., and Dangl, J. L. 2006. The plant immune system. Nature 444:323-329.

Kim, M. G., da Cunha, L., McFall, A. J., Belkhadir, Y., DebRoy, S., Dangl,
J. L., and Mackey, D. 2005. Two Pseudomonas syringae type III effectors inhibit RIN4-regulated basal defense in Arabidopsis. Cell 121:749759.

Lee, S. W., Han, S. W., Sririyanum, M., Park, C. J., Seo, Y. S., and Ronald, P. C. 2009. A type I-secreted, sulfated peptide triggers XA21-mediated innate immunity. Science 326:850-853.

Navarro, L., Zipfel, C., Rowland, O., Keller, I., Robatzek, S., Boller, T., and Jones, J. D. 2004. The transcriptional innate immune response to flg22. Interplay and overlap with Avr gene-dependent defense responses and bacterial pathogenesis. Plant Physiol. 135:1113-1128.

Park, J. H., Halitschke, R., Kim, H. B., Baldwin, I. T., Feldmann, K. A., and Feyereisen, R. 2002. A knock-out mutation in allene oxide synthase results in male sterility and defective wound signal transduction in Arabidopsis due to a block in jasmonic acid biosynthesis. Plant J. 31:112.

Ritter, C., and Dangl, J. L. 1996. Interference between two specific pathogen recognition events mediated by distinct plant disease resistance genes. Plant Cell 8:251-257.

Rutherford, S. L., and Lindquist, S. 1998. Hsp90 as a capacitor for morphological evolution. Nature 396:336-342.

Sato, M., Tsuda, K., Wang, L., Coller, J., Watanabe, Y., Glazebrook, J., and Katagiri, F. 2010. Network modeling reveals prevalent negative regulatory relationships between signaling sectors in Arabidopsis immune signaling. PLoS Pathog. 6:e1001011.

Shah, J. 2003. The salicylic acid loop in plant defense. Curr. Opin. Plant Biol. 6:365-371.

Tao, Y., Xie, Z., Chen, W., Glazebrook, J., Chang, H. S., Han, B., Zhu, T., Zou, G., and Katagiri, F. 2003. Quantitative nature of Arabidopsis responses during compatible and incompatible interactions with the bacterial pathogen Pseudomonas syringae. Plant Cell 15:317-330.

Tian, D., Traw, M. B., Chen, J. Q., Kreitman, M., and Bergelson, J. 2003. Fitness costs of $R$-gene-mediated resistance in Arabidopsis thaliana. Nature 423:74-77.

Tsuda, K., Sato, M., Stoddard, T., Glazebrook, J., and Katagiri, F. 2009. Network properties of robust immunity in plants. PLoS Genet. 5:e1000772.

Wildermuth, M. C., Dewdney, J., Wu, G., and Ausubel, F. M. 2001. Isochorismate synthase is required to synthesize salicylic acid for plant defence. Nature 414:562-565.

Zipfel, C., Robatzek, S., Navarro, L., Oakeley, E. J., Jones, J. D., Felix, G., and Boller, T. 2004. Bacterial disease resistance in Arabidopsis through flagellin perception. Nature 428:764-767.

Zipfel, C., Kunze, G., Chinchilla, D., Caniard, A., Jones, J. D., Boller, T., and Felix, G. 2006. Perception of the bacterial PAMP EF-Tu by the receptor EFR restricts Agrobacterium-mediated transformation. Cell $125: 749-760$. 